\title{
Synthesis, in silico study and in vitro anti-microbial evaluation of some new N-benzoyl-N'-[2-(4-chloro-phenoxy)-acetyl]-hydrazides analogs
}

\author{
Fares Hezam Al-Ostoot ${ }^{1,2}$, Yasser Hussein Eissa Mohammed ${ }^{1,3}, Z^{2}$ abiulla $^{1}$, Anup Nagaralli Kempaiah ${ }^{1}$, Shaukath Ara Khanum ${ }^{1 *}$ \\ ${ }^{1}$ Department of Chemistry, Yuvaraja's College, University of Mysore, Mysuru, India. \\ ${ }^{2}$ Department of Biochemistry, Faculty of Education and Science, Al-Baydha University, Yemen. \\ ${ }^{3}$ Department of Biochemistry, Faculty of Applied Science, University of Hajjah, Yemen.
}

\section{ARTICLE INFO \\ Received on: 30/12/2018 \\ Accepted on: 10/04/2019 \\ Available online: 01/07/2019}

Key words:

Phenoxyhydrazide, zone of

inhibition, in-silico study.

\begin{abstract}
In bacterial resistance duty, the prompt increase against existent anti-microbial drugs is a challenging universal health problem. Bacteria represent a highly significant threat globally to healthcare-associated infections, also responsible for the majority of hospital infections, which leads to an increase in the mortality and burden of worldwide healthcare duty. In this investigation, we reported the synthesis novel substitutes of phenoxy hydrazide analogs (6a-f) and have been screened for in vitro anti-bacterial and anti-fungal activities to determine the inhibition zone by using the paper disk agar diffusion technique and broth dilution to evaluate the minimum inhibitory concentration values. The structure-activity relationship suggest that among the series (6a-f), compounds (6e) with two chloro groups and (6f) with four fluoro groups showed good inhibition against pathogenic microbes. Furthermore, these results were also confirmed by the in silico study. Based on this studies, there are a scopes of developing compounds (6e) and (6f) into potent anti-microbial drugs in the near future.
\end{abstract}

\section{INTRODUCTION}

A pathogen infection is a microorganism that is able to cause most of the life-threatening disease and have become a serious public medical issue in the current days (Spellberg et al., 2008). Since there are huge interests in the up growth of the novel anti-microbial drugs future as a result of the gradual growth of bacterial and fungal resistance to a wide range of factors. Staphylococcus aureus is the main global, as well as extremely dangerous human pathogens that cause the variety of diseases, tend to range from fairly acute skin infections to deadly pneumonia and is one of the world's most serious causes of morbidity (Al-Ostoot et al., 2018; Arias and Murray, 2009; Perron et al., 2013).In addition, with the worrisome increase in multi-drug

"Corresponding Author

Shaukath Ara Khanum, Associate Professor, Department of Chemistry, Yuvaraja's College, University of Mysore, Mysore, India.

E-mail:shaukathara@yahoo.co.in resistant bacteria species, doctors are now ultimately obliged to refer patients to the second or even third antibiotic available option to combat the highly resistant of pathogens (Harrison and Svec, 1998). Hence, it is vitally important to reveal and develop new anti-microbial agents to combat these diseases (Vaikunthanathan et al., 2016). Natural products show to be the best source of new bioactive substrates in the design of anti-microbial pharmaceuticals; furthermore, their synthetic and semi-synthetic derivative products have also a wonderful clinical value as antibiotic agents (Dhami et al., 2014). The synthesized compounds with hydrazide moiety exhibited diverse pharmacological activities such as anti-oxidants (Bär et al., 2018), analgesic (Sheykhi-Estalkhjani et al., 2018), anti-bacterial (Gholivand et al., 2010; Morjan et al., 2014; Ozdemir et al., 2015), antifungal (Backes et al., 2014), anti-HIV (Yang et al., 2016), antituberculosis (Velezheva et al., 2016), anti-genotoxic (Bordoloi et al., 2009), anti-neoplastic drugs agents (Mohammed et al., 2017; 2018). Due to their wide potential uses as a medicinal drug, special attention has also been given to phenoxy acetic acid as well as its derivative products. In recent years, phenoxy hydrazides and 
the corresponding phenoxy acetic acid condensation derivatives draw as much attention throughout the field of synthetic and medicinal chemistry because of their different biological actions. Earlier our group has reported various compounds bearing phenoxy moiety as anti-microbial (Khanum et al., 2003), anti-oxidant (Prashanth et al., 2013), anti-inflammatory (Khanum et al., 2008) and anti-cancer agents (Kim et al., 2016; Puttaswamy et al., 2018; Zabiulla et al., 2016). As our ongoing work for a search of new drug (Kumar et al., 2018a; 2018b; Kumara et al., 2019), we have synthesis initially active phenoxy acetic hydrazide moiety (4) and then coupled with different substituted aromatic acids having an active group members, such as fluoro, chloro, bromo, and nitro to produce the phenoxy hydrazide analogs (6a-f, Fig. 1) and evaluated their anti-microbial activity.

\section{RESULTS AND DISCUSSION}

\section{Chemistry}

Scheme 1 outlined the synthesis reactions of the title compounds N-benzoyl-N'-[2-(4-chloro-phenoxy)-acetyl]hydrazide (6a-f). The compounds $(\mathbf{6 a}-\mathbf{f})$ were obtained by refluxing of 4-chloro phenol (1) with ethyl chloroacetate (2) in the presence of anhy. $\mathrm{K}_{2} \mathrm{CO}_{3}$ to give the desired ethyl-2-(4-chlorophenoxy) acetate (3) which to treat by hydrazine hydrate in the presence of ethanol as a solvent to afford (4-chloro-phenoxy)-acetic acid hydrazide (4). Finally, substituted benzoic acids (5a-f, $2.2 \mathrm{mmol})$ in the solvent of dichloromethane (DCM) $(25 \mathrm{ml})$ was stirred at $30^{\circ} \mathrm{C}$, and then the lutidine $(3 \mathrm{mmol})$ was added to the mixture, which then followed by adding of hydrazide compounds with (4, $2.2 \mathrm{mmol})$. The mixture of the reaction stirred for 20 minutes at $25^{\circ} \mathrm{C}-30^{\circ} \mathrm{C}$, and then, the environmental condition of reaction has been changed to cooled in $0^{\circ} \mathrm{C}-5^{\circ} \mathrm{C}, 2-(1 \mathrm{H}$-benzotriazole1-yl)-1,1,3,3 tetramethylammonium tetrafluoroborate (TBTU, 2 $\mathrm{mmol}$ ) was then added over a period of 20 minutes with keeping the temperature condition below than $5^{\circ} \mathrm{C}$. The reaction has been fully stirred for $13-15$ hours at the same condition. Thin layer chromatography (TLC) technique is often used to monitor the progress of chemical reactions and identify compounds present in a given mixture using mobile phase system of hexane: ethyl acetate (6:3). The remaining solvent was completely eliminated at reduced

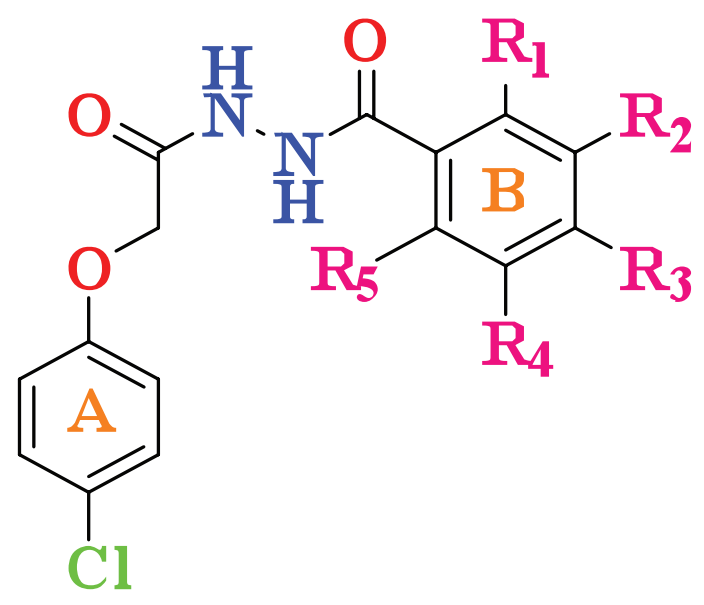

Figure 1. N-Benzoyl-N'-[2-(4-chloro-phenoxy)-acetyl]-hydrazides analogs. pressure by adding mashed ice to the mixture with stirring for a few minutes. The solid was filtered and recrystallized with ethanol to obtain compounds (6a-f) (Geetha et al., 2019). compounds structure of $\mathbf{6 a}-\mathbf{f}$ were confirmed by using some spectroscopy as nuclear magnetic resonance (NMR), infrared (IR), and liquid chromatography-mass spectrometry (LC-MS) techniques. The compound of ester (3) formation was confirmed by observing the disappearance of compound (1) $\mathrm{OH}$ stretching and the appearance of new carbonyl group stretching band in the IR absorption spectrum for the ester group at $1,670 \mathrm{~cm}^{-1}$. Compoundes proton for NMR spectrum can revealed by proton disappeared of broad singlet in the hydroxy group of phenol compound (1) and a triplet $\mathrm{CH}_{2}$ and quartet $\mathrm{CH}_{3}$ for and protons at $\delta 1.10$ and $\delta 4.13 \mathrm{ppm}$, respectively, was appeared in compound (3). In addition, the mass spectrum gave significant stable $\mathbf{M}^{+}$and $\mathbf{M}^{+2}$ peaks at $m / z 214$ and 216, respectively, which can clearly confirmed the ester of compound (3) formation. In the same way, the spectrum of the hydrazide compound (4) confirmed by the presentation of $\mathrm{NH}$ and $\mathrm{NH}_{2}$ stretching bands in the range of $3,100-3,205 \mathrm{~cm}^{-1}$ and $3,305-3,415 \mathrm{~cm}^{-1}$, respectively, in the IR spectrum. Furthermore, in the proton NMR of compound (4), the appearance of two singlet peaks $\mathrm{NH}_{2}$ and $\mathrm{NH}$ protons at $\delta 4.33$ and $\delta 9.80$ ppm, respectively, was confirmed. Also, the mass spectrum of compound (4) showed significant $\mathrm{M}^{+}$and $\mathrm{M}^{+2}$ peaks at $m / z 200$ and 202, respectively. Furthermore, (6a) compound spectrum was considered as a model to explain the spectral data for the $(\mathbf{6 a}-\mathbf{f})$ compounds that was easily revealed with respectively disappearance of $\mathrm{NH}_{2}$ and $\mathrm{COOH}$ stretching bands of the compounds (4) and (5a), and the appearance of $\mathrm{NH}$ stretching band at the range of 3,210-3,320 $\mathrm{cm}^{-1}$ in the IR spectrum. NMR spectrum was confirmed by the disappearance of $\mathrm{NH}_{2}$ proton of (4) and $\mathrm{COOH}$ protons of (5a) and appearance of $\mathrm{NH}$ proton peak at $\delta 10.71 \mathrm{ppm}$. Besides, there is an increase in aromatic protons in range of $\delta 6.98-8.20 \mathrm{ppm}$ and the mass spectrum also showed significant stable $\mathrm{M}^{+}$and $\mathrm{M}^{+2}$ peaks at $\mathrm{m} / z 384$ and 486, respectively, which clearly confirmed the formation of title product $(\mathbf{6 a})$.

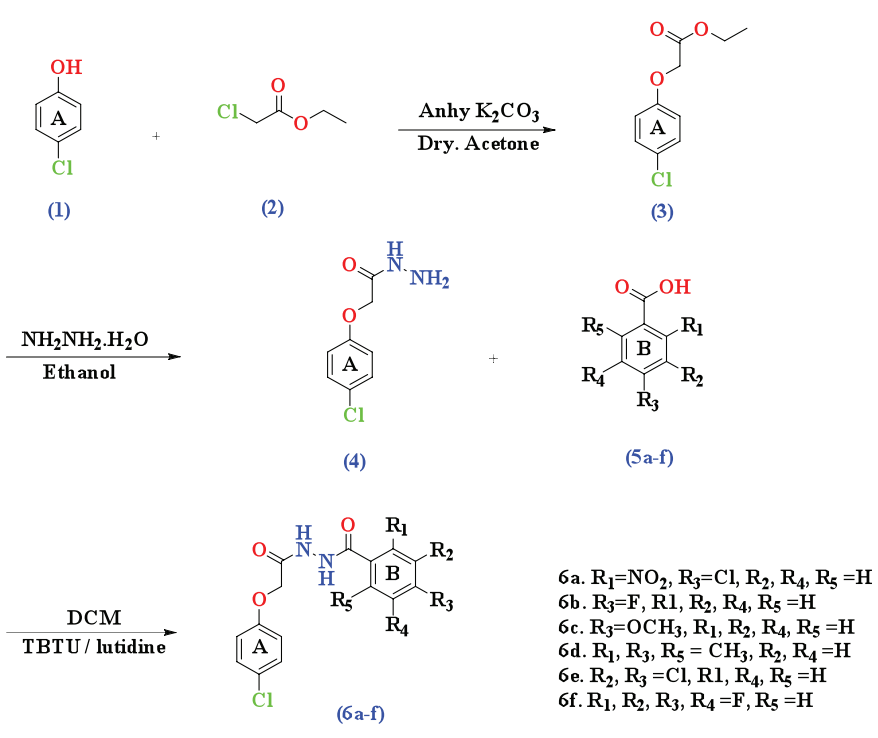

Scheme 1. Synthesis of novel N-benzoyl-N'-[2-(4-chloro-phenoxy)-acetyl]hydrazides analogs $(\mathbf{6 a}-\mathbf{f})$. 


\section{Biology}

The title compounds $(\mathbf{6} \mathbf{a}-\mathbf{f})$ were screened for their antimicrobial activity using the cup-plate method in the sabouraud agar medium. From anti-bacterial evaluation results against $B$. cereus, E. coli, $S$. aureus, and $P$. aeruginosa it is evident that most of the title compounds has exhibited comparable activity with reference to the standard compound ampicillin. The title compounds were also evaluated for their anti-fungal activity against $A$. niger, A. flavus, $R$. stolonifer, F. oxisporum using, and fluconazole as standard compound shown in Table 1. The zone of inhibition ( $\mathrm{mm}$ ) of each compound was determined and compared with the standard drugs. Compounds bearing two chloro groups at position 3 and 4 in ring $\mathbf{B}$ (6e) and four fluoro groups at position $2,3,4$, and 5 in ring $\mathbf{B}(\mathbf{6 f})$ were found to be highly active against both bacterial (Fig. 2) and fungal strain (Fig. 3). The results indicated that compounds bearing electron withdrawing groups exhibited good activity against both of the strains. Besides, the other compounds exhibited moderated anti-bacterial and antifungal activities. Furthermore, minimum fungicidal concentration (MFC), MIC, and finally minimum bacterial concentration (MBC) assays designed for all the synthesized compounds of (6a-f) were also carried out in which compounds (6e) and (6f) have shown less MIC, MBC, and MFC values compared to the other compounds shown in Tables 2 and 3. Thus, compounds of (6e) and (6f) can be used in near future for the treatment of pathogenic infection diseases.

\section{SAR study}

Through the review of literature which showed that the phenoxy acetic acid hydrazide and their derivatives has a potential pharmacological activity as anti-microbial, antioxidants, and anti-cancer. This study involves the multistep synthesis of phenoxy acetic acid hydrazide hybrids with phenyl acid derivatives Scheme 1. A new hydrazide derivativesNbenzoyl-N'-[2-(4-chloro-phenoxy)-acetyl]-hydrazides (6a-f), were synthesized, characterized and estimated for their anti- microbial actions against of four bacterial and four fungal strains. Compound (6e) bearing two chloro groups at positions 3 and 4 in ring $\mathbf{B}$ has shown slightly more activity compared to compound (6f) against all the four bacterial and fungal strains as shown in Table 1. For that reason, (6e) was chosen as a potent compound, based on its significant structure-activity relationship (SAR) and further verified by in-silico study docking with 1 kznprotein.

\section{Compound (6e) interacts with $1 \mathrm{kzn}$ protein in-silico}

By using the modeling study, compound (6e) was docked into the active site of the protein $1 \mathrm{kzn}$ subunit and then was analyzed for binding free energy and their interactions with the receptor. The ligand (6e) interacts through hydrogen bonding with the DNA-gyrase binding site. The distance of hydrogen bonds was shorter than $3.5 \AA$. The best conformations from the docking procedure with the best scored pose and the lowest binding energy $(-6.5 \mathrm{kcal} / \mathrm{mol})$. The results confirmed that the in-silico study was matched with in-vitro study as shown in Figure 4.

\section{MATERIALS AND METHODS}

All solvents and reagents, phenols substituted, ethyl chloro acetate, hydrazine hydrate, acids, 2,6 lutidine, and O-(benzotriazol-1-yl)-N,N, $\mathrm{N}^{\prime}, \mathrm{N}^{\prime}$-TBTU, were purchased from Sigma Aldrich Chemicals Pvt Ltd. with a purity of $90 \%$ $99 \%$. Analytical TLC was performed on $0.30 \mathrm{~mm}$ of silica gel plates (Merck 60 F 254 ) by using the solvent of ethyl acetate: hexane (2:7) system and visualized by UV-light. The melting point has been determined using a digital thermometer depending melting point device based on the Chemi Line CL725 microcontroller. NMR spectrum was recorded on a VNMRS-400 MHz Agilent-NMR spectrophotometer in CDCl3 or dimethyl sulfoxide (DMSO). The potassium bromide pellet method use to collected the IR spectrum on the Cary 630 FTIR Agilent spectrophotometer, VG70-70H spectrometer was used

Table 1. Anti-microbial activity of compounds (6a-f) (zone of inhibition, mm) against clinical pathogens.

\begin{tabular}{|c|c|c|c|c|c|c|c|c|c|}
\hline \multirow{2}{*}{\multicolumn{2}{|c|}{ Compounds }} & \multicolumn{4}{|c|}{ Bacterial strains } & \multicolumn{4}{|c|}{ Fungal strains } \\
\hline & & \multirow{2}{*}{$\frac{\text { B. cereus }}{11.3 \pm 0.31}$} & \multirow{2}{*}{$\frac{\text { E. coli }}{8.9 \pm 0.41}$} & \multirow{2}{*}{$\begin{array}{l}\text { S. aureus } \\
8.2 \pm 0.51\end{array}$} & \multirow{2}{*}{$\frac{P . \text { aeruginosa }}{10.6 \pm 0.25}$} & \multirow{2}{*}{$\begin{array}{c}\text { A. niger } \\
10.3 \pm 0.35\end{array}$} & \multirow{2}{*}{$\frac{\text { A. flavus }}{11.3 \pm 0.41}$} & \multirow{2}{*}{$\frac{R \text {. stolonifer }}{12.3 \pm 0.51}$} & \multirow{2}{*}{$\frac{\text { F. oxisporum }}{9.2 \pm 0.35}$} \\
\hline $6 a$ & ZI & & & & & & & & \\
\hline & $\mathrm{AI}$ & 0.463 & 0.705 & 0.430 & 0.493 & 0.584 & 0.662 & 0.802 & 0.425 \\
\hline \multirow[t]{2}{*}{$6 b$} & $\mathrm{ZI}$ & $12.6 \pm 0.52$ & $13.3 \pm 0.52$ & $14.3 \pm 0.40$ & $12.9 \pm 0.31$ & $18.5 \pm 0.54$ & $17.5 \pm 0.56$ & $15.2 \pm 0.91$ & $16.3 \pm 0.51$ \\
\hline & $\mathrm{AI}$ & 0.516 & 1.053 & 0.750 & 0.599 & 1.049 & 1.025 & 0.992 & 0.753 \\
\hline \multirow[t]{2}{*}{$6 c$} & $\mathrm{ZI}$ & $8.7 \pm 0.40$ & $10.1 \pm 0.34$ & $8.1 \pm 0.50$ & $9.1 \pm 0.34$ & $15.6 \pm 0.10$ & $12.1 \pm 0.48$ & $12.2 \pm 0.49$ & $14.6 \pm 0.81$ \\
\hline & $\mathrm{AI}$ & 0.356 & 0.799 & 0.424 & 0.422 & 0.724 & 0.708 & 0.795 & 0.674 \\
\hline \multirow[t]{2}{*}{$6 \mathrm{~d}$} & ZI & $8.9 \pm 0.31$ & $10.4 \pm 0.41$ & $7.9 \pm 0.49$ & $11.8 \pm 0.44$ & $9.7 \pm 0.31$ & $11.7 \pm 0.42$ & $12.1 \pm 0.46$ & $8.3 \pm 0.38$ \\
\hline & $\mathrm{AI}$ & 0.364 & 0.823 & 0.414 & 0.548 & 0.450 & 0.685 & 0.789 & 0.383 \\
\hline \multirow[t]{2}{*}{$6 e$} & $\mathrm{ZI}$ & $18.4 \pm 0.56$ & $19.6 \pm 0.65$ & $19.5 \pm 0.52$ & $22.3 \pm 0.42$ & $19.7 \pm 0.11$ & $21.5 \pm 0.32$ & $20.1 \pm 0.62$ & $20.9 \pm 0.11$ \\
\hline & AI & 0.549 & 1.552 & 1.023 & 1.036 & 1.117 & 1.260 & 1.311 & 0.873 \\
\hline \multirow[t]{2}{*}{$6 f$} & ZI & $17.6 \pm 0.43$ & $21.5 \pm 0.86$ & $16.6 \pm 0.13$ & $19.5 \pm 0.52$ & $20.1 \pm 0.62$ & $18.3 \pm 0.54$ & $18.8 \pm 0.52$ & $20.4 \pm 0.86$ \\
\hline & $\mathrm{AI}$ & 0.721 & 1.702 & 0.870 & 0.906 & 1.140 & 1.072 & 1.226 & 1.035 \\
\hline \multicolumn{2}{|c|}{ Ampicillin } & $24.4 \pm 0.53$ & $12.63 \pm 0.64$ & $19.07 \pm 0.33$ & $21.52 \pm 0.43$ & - & & - & - \\
\hline \multicolumn{2}{|c|}{ Flucanazole } & - & - & - & - & $21.52 \pm 0.63$ & $17.07 \pm 0.52$ & $15.33 \pm 0.84$ & $21.94 \pm 0.30$ \\
\hline
\end{tabular}

Where $\mathrm{AI}=$ activity index and $\mathrm{ZI}=$ zone of inhibition.

Standards: Flucanazole $(1.0 \mathrm{mg} /$ disc $)$, Ampicillin $(1.0 \mathrm{mg} /$ disc); AI- activity index $=$ ZI of test sample/ZI of standard. Values are mean of triplicate of the readings (mean $\pm \mathrm{SD}$ ). 


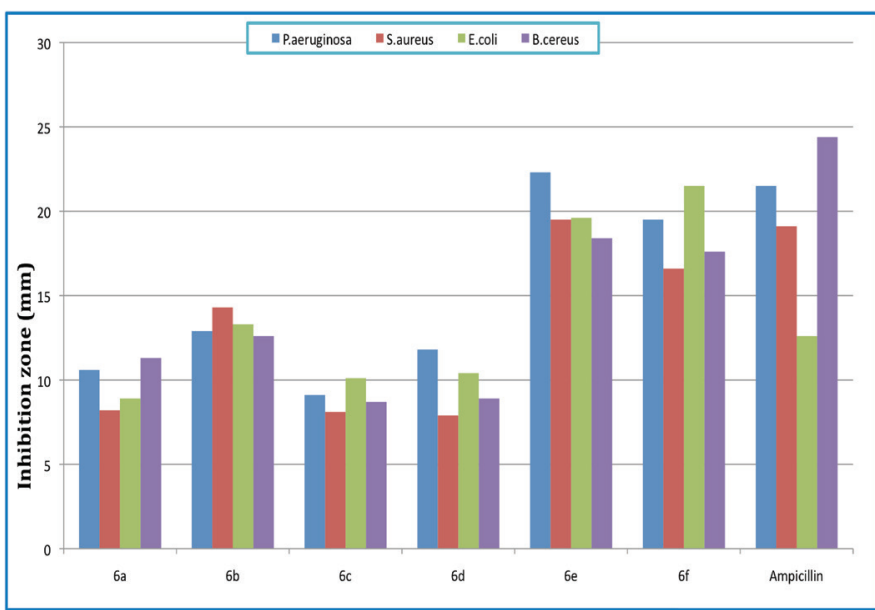

Figure 2. Represents that comparison among six compounds of N-benzoyl$\mathrm{N}^{\prime}$-[2-(4-chloro-phenoxy)-acetyl]-hydrazides (6a-f) analogs against bacterial strains.

to obtained Mass spectrum data. Elemental analysis results are within $0.5 \%$ of the calculated value.

\section{Chemistry}

General synthetic procedure for ethyl 2-(4-chlorophenoxy)acetate (3)

A reaction mixture of 4-chlorophenol (1, $0.030 \mathrm{~mol})$, ethylchloro acetate $(0.032 \mathrm{~mol})$ and potassium carbonate $(0.075$ mol, anhydrous) was refluxed in the solvent of dry acetone $(40 \mathrm{ml})$ for 8 hours to obtained phenoxy acetic ethyl ester (3). TLC technique used to monitor the completion of the reaction by using the solvent system of hexane: ethyl acetate $(3: 1)$. The mixture of the reaction was cooled and the solvent was removed by distillation method after completion of the reaction. To remove potassium carbonate, the residual mass was poured into cold water and extracted with ether $(3 \times 20 \mathrm{ml})$. The ether layer was washed with $10 \%$ of sodium hydroxide solution $(3 \times 20 \mathrm{ml})$, followed by water $(3 \times 20 \mathrm{ml})$,

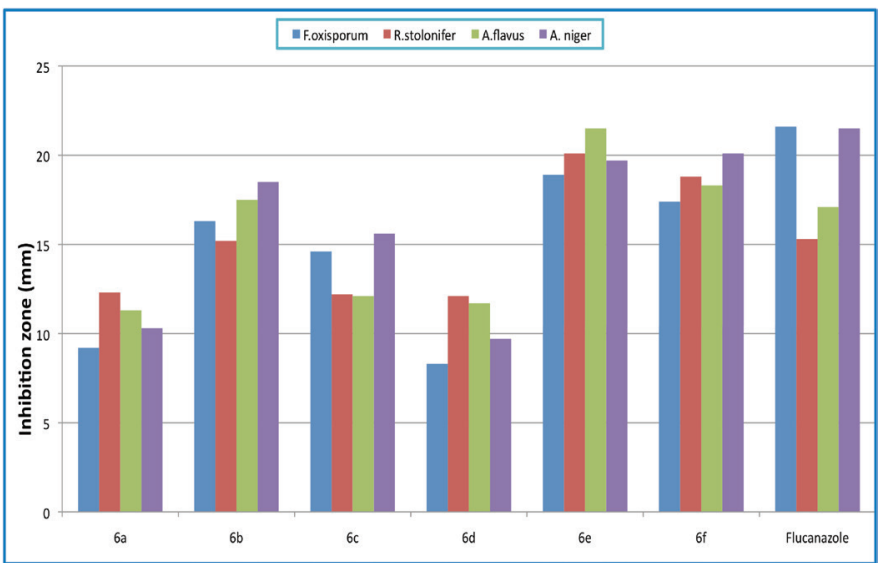

Figure 3. Represents that comparison among six compounds of N-benzoyl-N'[2-(4-chloro-phenoxy)-acetyl]-hydrazides (6a-f) analogs against fungal strains.

then to remove moisture, passed over anhydrous sodium sulfate and evaporated to obtained liquid ester compound (3).

Ethyl 2-(4-chlorophenoxy)acetate (3)

Yield $70 \%$; B.P. $177^{\circ} \mathrm{C}$; FT-IR (KBr) $v_{\max }\left(\mathrm{cm}^{\circ}\right)$ : 1,732-1,752 (ester, $\mathrm{C}=\mathrm{O}), 1,132$ (ester, C-O); ${ }^{1} \mathrm{HNMR}(400 \mathrm{MHz}$, DMSO-d $\left.{ }_{6}\right) \delta(\mathrm{ppm}): 1.19\left(t, 3 \mathrm{H}, \mathrm{CH}_{3}\right.$ of ester), $4.13\left(q, 2 \mathrm{H}, \mathrm{CH}_{2}\right.$ of ester), $4.76\left(\mathrm{~s}, 2 \mathrm{H}, \mathrm{OCH}_{2}\right), 6.67-6.88(\mathrm{~d}, 2 \mathrm{H}, \mathrm{J}=8 \mathrm{~Hz}, \mathrm{Ar}-\mathrm{H}), 7.41-$ $7.44(d, 2 H, J=8 \mathrm{~Hz}, \mathrm{Ar}-\mathrm{H})$; LC-MS m/z $214\left[\mathrm{M}^{+}\right], 216\left[\mathrm{M}^{+2}\right]$ (68), 128 (100), 184 (38), 186 (12), 170 (40), 170 (6). Anal.Calcd.for $\mathrm{C}_{10} \mathrm{H}_{11} \mathrm{ClO}_{3}$ (214): C, 55.96; H, 5.17. Found: $C, 55.87 \%$; $H, 5.13 \%$.

General synthetic procedure for (4-chloro-phenoxy)-acetic acid hydrazide (4)

The volume of $(0.018 \mathrm{~mol})$ hydrazine hydrate was added to a solution of compound (3) $(0.018 \mathrm{~mol})$ in absolute ethanol (25 $\mathrm{ml}$ ) and continuously stirred for 3-5 hours at room temperature. A solid was separated out, which was water quenched $(40 \mathrm{ml})$, filtered probably and then washed with water $(40 \mathrm{ml})$. Finally,

Table 2. MIC $(\mu \mathrm{g} / \mathrm{ml})$ and $\mathrm{MBC}$ performance of compounds (6a-f) against bacterial strains.

\begin{tabular}{|c|c|c|c|c|c|}
\hline \multirow{2}{*}{\multicolumn{2}{|c|}{ Compounds }} & \multicolumn{4}{|c|}{ Bacterial strains } \\
\hline & & \multirow{2}{*}{$\frac{\text { B. cereus }}{33.7}$} & \multirow{2}{*}{$\begin{array}{c}\text { E. coli } \\
47.8\end{array}$} & \multirow{2}{*}{$\frac{\text { S. aureus }}{49.3}$} & \multirow{2}{*}{$\frac{\text { P. aeruginosa }}{45.4}$} \\
\hline $6 a$ & MIC & & & & \\
\hline & MBC & 67.5 & 95.6 & 97.8 & 99.8 \\
\hline $6 \mathrm{~b}$ & MIC & 32.4 & 45.7 & 47.3 & 43.4 \\
\hline $6 \mathrm{c}$ & MBC & 98.3 & 100.3 & 99.8 & 99.8 \\
\hline \multirow[t]{2}{*}{$6 \mathrm{~d}$} & MIC & 39.9 & 55.5 & 50.8 & 48.5 \\
\hline & $\mathrm{MBC}$ & 78.9 & 112.4 & 101.9 & 88.7 \\
\hline $6 f$ & MBC & 45.8 & 78.3 & 85.9 & 93.1 \\
\hline Ampicillin & & 1.18 & 2.33 & 2.50 & 2.12 \\
\hline
\end{tabular}


Table 3. MIC ( $\mu \mathrm{g} / \mathrm{ml})$ and MFC performance of compounds (6a-f) against fungal strains.

\begin{tabular}{cccccc}
\hline \multirow{2}{*}{ Compounds } & \multicolumn{4}{c}{ Fungal strains } \\
\cline { 2 - 6 } & & A. niger & A. flavus & R. stolonifer & F. oxisporum \\
\hline 6a & MIC & 55.2 & 57.3 & 49.8 & 58.3 \\
& MFC & 113.4 & 114.6 & 99.6 & 116.6 \\
6b & MIC & 53.6 & 55.7 & 45.3 & 55.8 \\
& MFC & 110.8 & 111.4 & 90.7 & 110.6 \\
6c & MIC & 59.5 & 58.2 & 60.2 & 59.1 \\
& MFC & 110.5 & 109.6 & 120.5 & 109.4 \\
6d & MIC & 60.5 & 62.5 & 65.5 & 63.2 \\
& MFC & 120.8 & 121.5 & 124.5 & 121.5 \\
& MIC & 51.6 & 47.3 & 39.5 & 53.5 \\
& MFC & 103.2 & 94.6 & 79 & 107.1 \\
6f & MIC & 49.6 & 52.3 & 42.7 & 48.3 \\
& MFC & 98.4 & 104.6 & 95.4 & 96.6 \\
& & 2.33 & 1.16 & 4.67 & 2.82 \\
\hline \multirow{2}{*}{ Flucanazole } & & & &
\end{tabular}
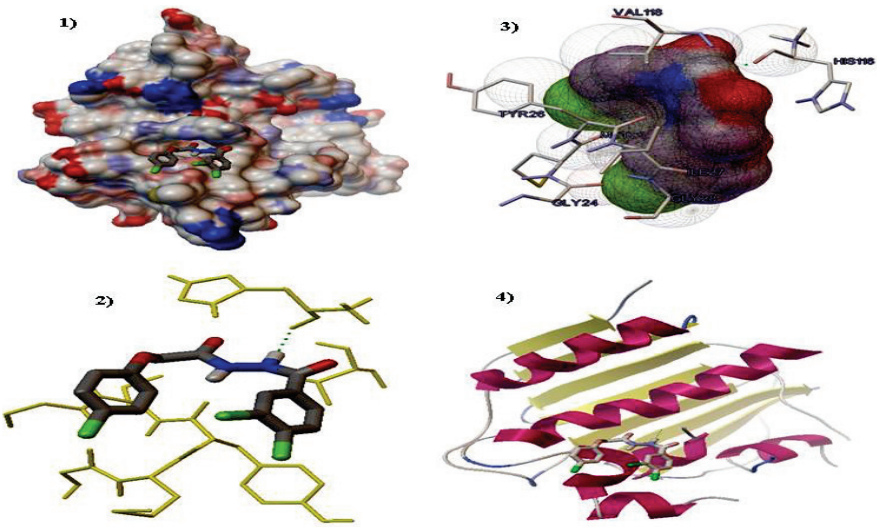

Figure 4. Compound (6e) interacts with $1 \mathrm{kzn}$ by binding with two amino acids HIS116 through hydrogen bond. (1) Enfolding of molecules (6e) in the active site $1 \mathrm{kzn}$ complexes. (2) Hydrogen bond interaction of molecule ligand (6e) with $1 \mathrm{kzn}$. (3) The 2D interaction analysis of (6e) with $1 \mathrm{kzn}$. (4) Ribbon models of $1 \mathrm{kzn}$ and ligand molecule (6e).

the solid has been dried under the vacuum and recrystallized with ethanol to obtain compound (4).

\section{(4-Chloro-phenoxy)-acetic acid hydrazide (4)}

Yield $78 \%$; M.P. $154^{\circ} \mathrm{C}-156^{\circ} \mathrm{C}$; FT-IR $(\mathrm{KBr}) v_{\max }\left(\mathrm{cm}^{-1}\right)$ : 1,670 (amide, $\mathrm{C}=\mathrm{O}), 3,122-3,220\left(\mathrm{NH}-\mathrm{NH}_{2}\right) ;{ }^{1} \mathrm{HNMR}(400 \mathrm{MHz}$, DMSO-d $) \delta$ (ppm): $4.33\left(s, 2 \mathrm{H}, \mathrm{NH}_{2}\right), 4.70\left(s, 2 \mathrm{H}, \mathrm{OCH}_{2}\right), 6.80$ $7.41(m, 4 H, \mathrm{Ar}-\mathrm{H}), 9.80(\mathrm{~s}, 1 \mathrm{H}, \mathrm{NH}) ; \mathrm{LC}-\mathrm{MS} \mathrm{m} / \mathrm{z} 200\left[\mathrm{M}^{+}\right], 202$ $\left[\mathrm{M}^{+2}\right]$ (75), 128 (100), 184 (28), 186 (12), 170 (6). Anal.Calcd. for $\mathrm{C}_{8} \mathrm{H}_{9} \mathrm{ClN}_{2} \mathrm{O}_{2}(200)$ : $C, 47.88 ; H, 4.50 ; N, 13.95$. Found: $C$, $47.82 \% ; H, 4.44 \% ; N, 13.90 \%$.

General synthetic procedure for benzoic acid N'-[2-(4-chlorophenoxy)-acetyl]-hydrazides (6a-f)

To the compound (4), in dry DCM, $(15 \mathrm{ml})$, substituted acids (5a-f) were added, followed by the addition of the of lutidine ( 1.2 vol.), at $25^{\circ} \mathrm{C}-30^{\circ} \mathrm{C}$. The reaction mixture was stirred at the same temperaturefor 20 minutes. The reaction was cooled to $0^{\circ} \mathrm{C}-5^{\circ} \mathrm{C}$ by using an ice bath, TBTU $(0.002 \mathrm{~mol})$ was added over a period of 25 minutes while maintaining the temperature condition below than $5^{\circ} \mathrm{C}$. The reaction was stirred overnight and in during TLC used for monitoring the progress with hexane: ethyl acetate $(3: 1)$ system. Then the reaction mixture was diluted with $(35 \mathrm{ml})$ of DCM and treated with $10 \%$ of sodium bicarbonate solution $(35 \mathrm{ml})$. The organic layer was washed with water $(35$ $\mathrm{ml}$ ), dried over anhydrous sodium sulfate and concentrated to yield compounds $(\mathbf{6 a}-\mathbf{f})$.

$N$-(4-chloro-2-nitro)benzoyl $\quad-N^{\prime}-[2-(4-c h l o r o-p h e n o x y)$-acetyl]hydrazide (6a)

Yield $65 \%$; M.P. $176^{\circ} \mathrm{C}-178^{\circ} \mathrm{C}$; FT-IR $(\mathrm{KBr}) v_{\max }\left(\mathrm{cm}^{-1}\right)$ : 1,645 (amide, $\mathrm{C}=\mathrm{O}), 3,125-3,220(\mathrm{NH}-\mathrm{NH}) ;{ }^{1} \mathrm{HNMR}(400 \mathrm{MHz}$, DMSO-d $) \delta(\mathrm{ppm}): 4.64\left(\mathrm{~s}, 2 \mathrm{H}, \mathrm{OCH}_{2}\right), 6.98-8.20(m, 7 \mathrm{H}, \mathrm{Ar}-\mathrm{H})$, $10.50(s, 1 H, \mathrm{NH}), 10.71(s, 1 H, \mathrm{NH}) ; \mathrm{LC}-\mathrm{MS} \mathrm{m} / \mathrm{z} 384\left[\mathrm{M}^{+}\right], 386$ $\left[\mathrm{M}^{+2}\right], 388\left[\mathrm{M}^{+4}\right]$ (100), 128 (6), 170 (35), 335 (35). Anal.Calcd. for $\mathrm{C}_{15} \mathrm{H}_{11} \mathrm{C}_{12} \mathrm{~N}_{3} \mathrm{O}_{5}$ (384): $C, 46.92 ; H, 2.89 ; N, 10.94$. Found: $C$, $46.85 \% ; H, 2.80 \% ; N, 10.90 \%$.

N-(4-fluoro)benzoyl- N'-[2-(4-chloro-phenoxy)-acetyl]-hydrazide (6b)

Yield $80 \%$; M.P. $173^{\circ} \mathrm{C}-175^{\circ} \mathrm{C}$; FT-IR (KBr) $v_{\text {max }}$ $\left(\mathrm{cm}^{-1}\right)$ : 1,645 (amide, $\left.\mathrm{C}=\mathrm{O}\right), 3,125-3,220(\mathrm{NH}-\mathrm{NH}) ;{ }^{1} \mathrm{HNMR}(400$ MHz, DMSO-d $) \delta(\mathrm{ppm}): 4.60\left(s, 2 \mathrm{H}, \mathrm{OCH}_{2}\right), 6.95-8.15(\mathrm{~m}, 7 \mathrm{H}$, Ar-H), $10.55(s, 1 H, \mathrm{NH}), 10.70(s, 1 H, \mathrm{NH}) ; \mathrm{LC}-\mathrm{MS} \mathrm{m} / \mathrm{z} 322$ $\left[\mathrm{M}^{+}\right], 324\left[\mathrm{M}^{+2}\right]$ (100), 128 (6), 170 (35), 335 (35). Anal.Calcd. for $\mathrm{C}_{15} \mathrm{H}_{12} \mathrm{ClFN}_{2} \mathrm{O}_{3}$ (322): $C, 55.83 ; H, 3.75 ; N, 8.68$. Found: $C$, $55.80 \% ; H, 3.70 \% ; N, 8.60 \%$.

$N$-(4-methoxy)benzoyl- N'-[2-(4-chloro-phenoxy)-acetyl]-hydrazide (6c)

Yield $74 \%$; M.P. $170^{\circ} \mathrm{C}-172^{\circ} \mathrm{C}$; FT-IR $(\mathrm{KBr}) v_{\max }\left(\mathrm{cm}^{-1}\right)$ : 1,640 (amide, $\mathrm{C}=\mathrm{O}), 3,125-3,220(\mathrm{NH}-\mathrm{NH}) ;{ }^{1} \mathrm{HNMR}(400 \mathrm{MHz}$, DMSO-d $\left.{ }_{6}\right) \delta(\mathrm{ppm}): 4.10\left(s, 3 H, \mathrm{OCH}_{3}\right), 4.65\left(s, 2 \mathrm{H}, \mathrm{OCH}_{2}\right)$, 6.90-8.19 ( $m, 8 H, \mathrm{Ar}-\mathrm{H}), 10.50(s, 1 H, \mathrm{NH}), 10.75(s, 1 H, \mathrm{NH})$; LC-MS m/z $334\left[\mathrm{M}^{+}\right], 336\left[\mathrm{M}^{+2}\right]$ (100), 128 (6), 170 (35), 335 (35). Anal. Calcd. for $\mathrm{C}_{16} \mathrm{H}_{15} \mathrm{ClN}_{2} \mathrm{O}_{4}$ (334): $C, 57.41 ; H, 4.52 ; N$, 8.37. Found: $C, 57.35 \% ; H, 4.50 \% ; \mathrm{N}, 8.30 \%$. 
$N$-(2,4,6-trimethyl)benzoyl- N'-[2-(4-chloro-phenoxy)-acetyll-hydrazide (6d)

Yield $74 \%$; M.P. $180^{\circ} \mathrm{C}-182^{\circ} \mathrm{C}$; FT-IR $(\mathrm{KBr}) v_{\max }\left(\mathrm{cm}^{-1}\right)$ : 1,640 (amide, $\mathrm{C}=\mathrm{O}), 3,125-3,220$ (NH-NH); ${ }^{1} \mathrm{HNMR}(400 \mathrm{MHz}$, DMSO-d $\left.{ }_{6}\right) \delta(\mathrm{ppm}): 2.66\left(s, 9 H, 3 \mathrm{CH}_{3}\right), 4.60\left(s, 2 \mathrm{H}, \mathrm{OCH}_{2}\right), 6.95$ $8.25(m, 6 H, \mathrm{Ar}-\mathrm{H}), 10.50(s, 1 H, \mathrm{NH}), 10.70(s, 1 H, \mathrm{NH}) ; \mathrm{LC}-\mathrm{MS}$ $\mathrm{m} / \mathrm{z} 346\left[\mathrm{M}^{+}\right], 348\left[\mathrm{M}^{+2}\right]$ (100), 128 (6), 170 (35), 335 (35). Anal. Calcd.for $\mathrm{C}_{18} \mathrm{H}_{19} \mathrm{ClN}_{2} \mathrm{O}_{3}$ (346): $C, 62.34 ; H, 5.52 ; N$, 8.08. Found: $C, 62.30 \% ; H, 5.50 \%$; N, $8.00 \%$.

$N$-(3,4-dichloro)benzoyl- $N^{\prime}$-[2-(4-chloro-phenoxy)-acetyl]hydrazide (6e)

Yield $65 \%$; M.P. $173^{\circ} \mathrm{C}-175^{\circ} \mathrm{C}$; FT-IR $(\mathrm{KBr}) v_{\max }\left(\mathrm{cm}^{-1}\right)$ : 1,640 (amide, $\mathrm{C}=\mathrm{O}), 3,125-3,220(\mathrm{NH}-\mathrm{NH}) ;{ }^{1} \mathrm{HNMR}(400 \mathrm{MHz}$, DMSO-d $\left.{ }_{6}\right) \delta(\mathrm{ppm}): 4.62\left(\mathrm{~s}, 2 \mathrm{H}, \mathrm{OCH}_{2}\right), 6.95-8.20(\mathrm{~m}, 7 \mathrm{H}, \mathrm{Ar}-\mathrm{H})$, $10.54(s, 1 H, \mathrm{NH}), 10.75(s, 1 H, \mathrm{NH}) ; \mathrm{LC}-\mathrm{MS} \mathrm{m} / \mathrm{z} 373\left[\mathrm{M}^{+}\right], 375$ $[\mathrm{M}+2], 377[\mathrm{M}+4], 379[\mathrm{M}+6](100), 128(6), 170(35), 335$ (35). Anal.Calcd.for $\mathrm{C}_{15} \mathrm{H}_{11} \mathrm{Cl}_{3} \mathrm{~N}_{2} \mathrm{O}_{3}$ (373): $C$, 48.22; $H, 2.97 ; \mathrm{N}$, 7.50. Found: $C, 48.20 \% ; H, 2.92 \% ; N, 7.45 \%$.

$N-\left(2,3,4,5-\right.$ Ttetrafluoro)benzoyl- $N^{\prime}-[2-(4-c h l o r o-p h e n o x y)$-acetyl]hydrazide (6f)

Yield $75 \%$; B.P. $182^{\circ} \mathrm{C}$; FT-IR $(\mathrm{KBr}) v_{\max }\left(\mathrm{cm}^{-1}\right): 1,640$ (amide, $\mathrm{C}=\mathrm{O}), 3,125-3,220$ (NH-NH); ${ }^{1} \mathrm{HNMR}(400 \mathrm{MHz}$, DMSO-d $\left._{6}\right) \delta(\mathrm{ppm}): 4.66\left(s, 2 \mathrm{H}, \mathrm{OCH}_{2}\right), 6.92-8.22(\mathrm{~m}, 5 \mathrm{H}, \mathrm{Ar}-$ H), $10.52(s, 1 \mathrm{H}, \mathrm{NH}), 10.70(s, 1 \mathrm{H}, \mathrm{NH}) ; \mathrm{LC}-\mathrm{MS} \mathrm{m} / \mathrm{z} 376[\mathrm{M}+]$, $378[\mathrm{M}+2](100), 128$ (6), 170 (35), 335 (35). Anal.Calcd.for $\mathrm{C}_{15} \mathrm{H}_{9} \mathrm{ClF}_{4} \mathrm{~N}_{2} \mathrm{O}_{3}$ (376): $C, 47.83 ; H, 2.41 ; N, 7.44$. Found: $C$, $47.80 \% ; H, 2.40 \% ; N, 7.40 \%$.

\section{Biological study}

Maintenance, culture, and preservation of the microorganisms

Cultures in the experimental parts of bacteria and fungi were obtained from the Department of Microbiology, Maharani College, Mysore University. Preparation appropriate culture media is one of the basics to study them. Maintained pure bacterial cultures were done on the nutrient agar medium and other fungal cultures were done on potato dextrose agar (PDA) medium. Further maintained were by sub-culturing all stock cultures regularly on the same of medium and incubated at $25^{\circ} \mathrm{C}-30^{\circ} \mathrm{C}$ for $7-8$ days and then stored at $4^{\circ} \mathrm{C}$ before to use in experiments.

\section{Media preparation and its sterilization}

Anti-microbial susceptibility has been evaluated in Petri dishes by using agar well diffusion methods on solid (Agar-Agar) media (Yehia et al., 2013). Nutrient agar (NA) (34 $\mathrm{g} / \mathrm{l}$ ) and PDA (34 g/l) were used for the bacterial assay to develop the surface of the colony growth. The MBC, MFC and MIC, values were evaluated using the serial microdilution assays. Suspension culture, for bacterial cells growth, was done by preparing $2.25 \%$ Lauria Broth (w/v), and for the fungus cells growth, $2.45 \%(\mathrm{w} / \mathrm{v})$ Potato dextrose broth (PDB) was taken for an estimate. Sterilized all the prepared media by autoclaving at $\left(122^{\circ} \mathrm{C}\right)$ for 25 minutes.

\section{Microbiological screening}

Agar well diffusion method was used to evaluation antimicrobial activities of different compounds, and MIC (Nwakanma et al., 2016).
Agar well diffusion method

Agar well-diffusion method has been used to evaluate anti-microbial activity. Both the NA and PDA plates have been swabbed (sterile cotton swabs) with a 7 hours old - broth culture of the various different bacteria and fungi. Wells $(10 \mathrm{~mm}$ diameter as well as about $2 \mathrm{~cm}$ in a part) have been probably made in all of these plates using sterile cork borer. Stock solution was prepared for each six compounds at a concentration of $1 \mathrm{mg} / \mathrm{ml}$. Approximately $100 \mu \mathrm{l}$ of different of compound concentrations were added to the wells with a sterile syringe and allowed to spread for 2 hours at $25^{\circ} \mathrm{C}$. Control experiments were arranged that included inoculums without compounds. The plates have been incubated for bacterial pathogens at $38^{\circ} \mathrm{C}$ for $198-24$ hours and for fungal pathogens at $27^{\circ} \mathrm{C}$ for 45 hours. Zone inhibition $(\mathrm{mm})$ diameter was measured and the activity index had been calculated. Test was constantly repeated for three times (Triplicates), and the measurements were taken in three totally different fixed directions for each replicate and the average values given were recorded (Sokovic et al., 2008).

\section{Preparation of inoculums}

Antibacterial activity test:

The anti-bacterial assays were performed using the micro-dilution test to determine the anti-bacterial activity of organic compounds evaluated against some of the pathogenic bacteria. adjusted of bacterial suspensions to a concentration of $1.0 \times 10^{7} \mathrm{CFU} / \mathrm{ml}$ was with sterile saline. The inocula were prepared, vacuum packaged and stored until use at $4^{\circ} \mathrm{C}$. Inocula dilutions have been grown on a solid type of media to confirm the absence of toxic effects, contamination and to check the validity of the inoculum buffer. All the experiments had all been duplicated and repeated three times constantly (Mimica-Dukić et al., 2003).

Test for anti-fungal activity:

A modified micro-dilution technique has been used to thoroughly investigate the compounds as anti-fungal activity. sterile of $0.85 \%$ saline containing $0.1 \%$ between $80(\mathrm{v} / \mathrm{v})$ was used to wash the fungal spores from the plates agar surface. The concentration of sterile saline $1.0-10^{7}$ was adjusted to spore suspension in a final volume of the $100 \mu \mathrm{l}$ per well. The inocula was stored to be used again at $4^{\circ} \mathrm{C}$. Serial dilutions of that same inoculum were cultured on solid potato dextrose agar to check both the validity of inoculum and the absence of contamination (Sen and Batra, 2012).

\section{Determination of MIC:}

A serial dilution method was used to perform the MBCs, MICs, and MFCs by using 96-well microtiter plates. Both for bacterial culture and fungus, potato dextrose broth medium with particular inoculum was used for the different six targeted compounds solution $(1 \mathrm{mg} / \mathrm{ml})$ with Luria broth for 72 hours, microplates were incubated at $25^{\circ} \mathrm{C}$, respectively. In the binocular microscope, MICs were defined as the lowest concentrations without visible growth (Omar et al., 2010).

\section{Determination of MBC:}

By serial subcultivation of $2 \mu$ into microtitre plates containing $100 \mu \mathrm{l}$ of broth per well, MBCs were determined 
for further incubation with 75 hours. MBCs were defined at the lowest concentration without visible growth which, indicating the original inoculum killed with 99\%. Microplate reader (Perlong, ENM8602) at $650 \mathrm{~nm}$ used to determine the of each well optical density and compared with bacterial ampicillin standards (Himedia lab, India) as the positive control. All experiments have been duplicated and repeated three times.

\section{Determination of MFC:}

By serial subcultivation of $2 \mu$ into Microtiter plates with $100 \mu \mathrm{l}$ of broth per well, the fungicidal concentrations (MFCs) were evaluated for the further incubated 75 hours at $25^{\circ} \mathrm{C}$. MFC indicating $99.5 \%$ killing of the original inoculum described as the lowest concentration with no visible growth, fluconazole has been used as a positive control for fungus with $(1-3,000 \mu \mathrm{g} / \mathrm{ml})$. All experiments have been duplicated and repeated three times.

\section{Molecular docking studies}

In order to predict the interaction of synthesized compounds with the binding sites of DNA-gyrase, molecular docking studies were performed. The crystal structure of the enzyme (PDB code $1 \mathrm{KZN}$ ) with resolution $4 \AA$ was chosen as the protein model for the current study. The structure of ligand was optimized using the BioChemUltra2010 as per the reported method. Molecules and parameters used by Auto Dock Tools were prepared before submitting for docking analysis. Polar hydrogen atoms have been added while non-polar hydrogen atoms were merged and then, Gasteiger partial atomic charges were assigned to the ligands. All rotatable ligand bonds, defined by the program's default, were allowed to rotate throughout the automated docking system and will then prepared protein and ligand structures were calculating of energy grid maps after saved in the PDBQT suitable format. A grid box size of $44 \times 44 \times 44 \AA$ points with a grid spacing of $0.377 \AA$ was considered. Lamarckian genetic algorithm program with an adaptive whole method search in the Auto Dock was chosen to calculate the conformers of different ligand (Yasser et al., 2018).

\section{CONCLUSION}

In conclusion, six novel hydrazide derivatives, N-benzoyl-N'-[2-(4-chloro-phenoxy)-acetyl]-hydrazides (6a-f), were synthesis, and evaluated for their anti-microbial action against four bacterial and four fungal strains. From the antimicrobial data, it was concluded that compounds $(\mathbf{6 e})$ bearing two chloro group and (6f) with four flouro group, at 3, 4 and 2, 3, 4, 5 positions, respectively in ring $\mathbf{B}$ were highly active against both of bacterial and fungal strains. Moreover, the two potent compounds (6e, 6f), showed good potential of MIC, MBC, and MFC among of all synthesis scheme. Furthermore, the in silico study results confirmed that the potent compound (6e), has good binding energy with exact pocket side to the specific protein and this result was matched to in vitro study.

\section{ACKNOWLEDGMENTS}

Fares Hezam Al-Ostoot is thankful to the government of Yemen and Al-Baydha University, Yemen, for providing financial assistance under the teacher's fellowship and Mysore University, Mysore, India. Yasser Hussein Eissa Mohammed is thankful to the University of Hajjah, Yemen, for providing financial assistance under the teacher's fellowship. Zabiulla gratefully acknowledges the financial support provided by the Department of Science and Technology, New Delhi, Under INSPIRE-Fellowship scheme [IF140407]. Shaukath Ara Khanum thankfully acknowledges the financial support provided by VGST, Bangalore, under CISEE Program [Project sanction order: No. VGST/CISEE/282].

\section{REFERENCES}

Al-Ostoot FH, Vidya R, Mohammed YH, Jyothi M, Pallavi HM, Khanum SA. Statistical analysis of antimicrobial data of 2-[2-(Aroyl) aroyloxy] methyl1, 3, 4 Oxadiazoles analogues using ANOVA. Asian J Res Chem, 2018; 11(2):293-7.

Arias CA, Murray BE. Antibiotic-resistant bugs in the 21st century - a clinical super-challenge. N Engl J Med, 2009; 360(5):439-43.

Backes GL, Neumann DM, Jursic BS. Synthesis and antifungal activity of substituted salicylaldehyde hydrazones, hydrazides and sulfohydrazides. Bioorgan Med Chem, 2014; 22(17):4629-36.

Bär F, Hopf H, Knorr M, Krahl J. Rancimat and PetroOxy oxidation stability measurements of rapeseed oil methyl ester stabilized with hydrazides and antioxidants. Fuel, 2018; 232:108-13.

Bordoloi M, Kotoky R, Mahanta JJ, Sarma TC, Kanjilal PB. Anti-genotoxic hydrazide from Crinum defixum. Eur J Med Chem, 2009; 44(6):2754-7.

Dhami NK, Reddy MS, Mukherjee A. Application of calcifying bacteria for remediation of stones and cultural heritages. Front Microbiol, 2014; 5:304.

Geetha DV, Al-Ostoot FH, Mohammed YH, Sridhar MA, Khanum SA, Lokanath NK. Synthesis, Elucidation, Hirshfeld surface analysis, and DFT calculations of 4-chloro-N-[2-(2-1H-indol-3-ylacetylamino)-phenyl]-benzamide. J Mol Struct, 2019; 1178:384-93.

Gholivand K, Hosseini Z, Farshadian S, Naderi-Manesh H. Synthesis, characterization, oxidative degradation, antibacterial activity and acetylcholinesterase /butyrylcholin esterase inhibitory effects of some new phosphorus (V) hydrazides. Eur J Med Chem, 2010; 45(11):5130-9.

Harrison JW, Svec TA. The beginning of the end of the antibiotic era? Part I. The problem: abuse of the "miracle drugs". Quintessence Int, 1998; 29(3)

Khanum SA, Khanum NF, Shashikanth M. Synthesis and antiinflammatory activity of 2-aryloxy methyl oxazolines. Bioorgan Med Chem Lett, 2008; 18(16):4597-601.

Khanum SA, Shashikanth S, Sudha BS. A facile synthesis and antimicrobial activity of 3-(2-aroylaryloxy) methyl-5-mercapto-4-phenyl4H-1, 2, 4-triazole and 2-(2-aroylaryloxy) methyl-5-N-phenylamino-1, 3, 4-thiadiazole analogues. Sci Asia, 2003; 34:383-92.

Kim M, Lee S, Park EB, Kim KJ, Lee HH, Shin JS, Fischer K, Koeberle A, Werz O, Lee KT, Lee JY. Hit-to-lead optimization of phenylsulfonyl hydrazides for a potent suppressor of PGE2 production: synthesis, biological activity, and molecular docking study. Bioorgan Med Chem Lett, 2016; 26(1):94-9.

Kumar SM, Hezam AO, Manjunath BC, Shamprasad VR, Mohammed YH, Mahesh N, Shaukath AK, Lokanath NK, Byrappa K. Crystal packing analysis of 1-(3, 4-dimethoxyphenyl)-3-(4-bromophenyl) prop-2-en-1-one exhibiting a putative halogen bond CBr O. J Mol Struct, 2018a; 1156:216-23.

Kumar SM, Manjunath BC, Al-Ostoot FH, Jyothi M, AlGhorbani M, Khanum SA, Kudva AK, Lokanath NK, Byrappa K. Synthesis, crystal structure and Hirshfeld surfaces of 1-(3, 4-dimethoxyphenyl)-3-(3hydroxyphenyl) prop-2-en-1-one. Chem Data Collect, 2018b; 15:153-60.

Kumara K, Al-Ostoot FH, Mohammed YH, Khanum SA, Lokanath NK. Synthesis, crystal structure and 3D energy frameworks of ethyl 2-[5-nitro-2-oxopyridine-1 (2H)-yl] acetate: Hirshfeld surface analysis and DFT calculations. Chem Data Collect, 2019; 6:100195.

Mimica-Dukić N, Kujundžić S, Soković M, Couladis M. Essential oil composition and antifungal activity of Foeniculum vulgare 
Mill. obtained by different distillation conditions. Phytother Res, 2003; 17(4):368-71.

Mohammed YH, Khanum SA. The critical role of novel benzophenone analogs on tumor growth inhibition targeting angiogenesis and apoptosis. MedChemComm, 2018; 9(4):639-56.

Mohammed YH, Malojirao VH, Thirusangu P, Al-Ghorbani M, Prabhakar BT, Khanum SA. The Novel 4-Phenyl-2-Phenoxyacetamide Thiazoles modulates the tumor hypoxia leading to the crackdown of neoangiogenesis and evoking the cell death. Eur J Med Chem, 2018; $143: 1826-39$

Mohammed YH, Thirusangu P, Vigneshwaran V, Prabhakar BT, Khanum SA. The anti-invasive role of novel synthesized pyridazine hydrazide appended phenoxy acetic acid against neoplastic development targeting matrix metallo proteases. Biomed Pharmacother, 2017; 95:375-86.

Morjan RY, Mkadmh AM, Beadham I, Elmanama AA, Mattar MR, Raftery J, Pritchard RG, Awadallah AM, Gardiner JM. Antibacterial activities of novel nicotinic acid hydrazides and their conversion into $\mathrm{N}$-acetyl-1, 3, 4-oxadiazoles. Bioorgan Med Chem Lett, 2014; 24(24):5796800 .

Nwakanma C, Njoku EN, Pharamat T. Antimicrobial activity of secondary metabolites of fungi isolated from leaves of bush mango. Next Generat Sequenc Appl, 2016; 3:135.

Omar K, Geronikaki A, Zoumpoulakis P, Camoutsis C, Soković M, Cirić A, Glamočlija J. Novel 4-thiazolidinone derivatives as potential antifungal and antibacterial drugs. Bioorgan Med Chem, 2010; 18(1):42632.

Ozdemir UO, Ilbiz F, Gunduzalp AB, Ozbek N, Genç ZK, Hamurcu F, Tekin S. Alkyl sulfonic acide hydrazides: synthesis, characterization, computational studies and anticancer, antibacterial, anticarbonic anhydrase II (hCA II) activities. J Mol Struct, 2015; 1100:464 74

Perron GG, Kryazhimskiy S, Rice DP, Buckling A. Multidrug therapy and evolution of antibiotic resistance: when order matters. Appl Environ Microbiol, 2012; 78(17):6137-42.

Prashanth T, Ranganatha VL, Naveen P, Gurupadaswamy HD, Begum AB, Al-Ghorbani M, Khanum SA. Synthesis of (4-benzoylphenoxy)-acetic acid derivatives and their efficacy as antioxidant agents Free Radicals Antioxidants, 2013; 3:S50-54.

Puttaswamy N, Malojiao VH, Mohammed YH, Sherapura A, Prabhakar BT, Khanum SA. Synthesis and amelioration of inflammatory paw edema by novel benzophenone appended oxadiazole derivatives by exhibiting cyclooxygenase-2 antagonist activity. Biomed Pharmacother, 2018; 103:1446-55.

Sen A, Batra A. Evaluation of antimicrobial activity of different solvent extracts of medicinal plant: Melia azedarach L. Int J Curr Pharm Res, 2012; 4(2):67-73.

Sheykhi-Estalkhjani A, Mahmoodi NO, Yahyazadeh A, Nadamani MP. Synthesis of new bis-benzylidene-hydrazides as a sensitive chromogenic sensor for naked-eye detection of $\mathrm{CN}^{-}$and $\mathrm{AcO}^{-}$ions. Tetrahedron, 2018; 74(37):4868-74.

Sokovic M, Marin PD, Brkic D, van Griensven LJ. Chemical composition and antibacterial activity of essential oils against human pathogenic bacteria. Food 2008; 1(2):220-6.
Spellberg B, Guidos R, Gilbert D, Bradley J, Boucher HW, Scheld WM, Bartlett JG, Edwards J Jr; Infectious Diseases Society of America. The epidemic of antibiotic-resistant infections: a call to action for the medical community from the Infectious Diseases Society of America. Clin Infect Dis, 2008; 46(2):155-64.

Vaikunthanathan T, Safinia N, Lombardi G, Lechler RI. Microbiota, immunity and the liver. Immunol Lett, 2016; 171:36-49.

Velezheva V, Brennan P, Ivanov P, Kornienko A, Lyubimov S, Kazarian K, Nikonenko B, Majorov K, Apt A. Synthesis and antituberculosis activity of indole-pyridine derived hydrazides, hydrazide-hydrazones, and thiosemicarbazones. Bioorgan Med Chem Lett, 2016; 26(3):978-85.

Yang L, Wang P, Wu JF, Yang LM, Wang RR, Pang W, Li YG, Shen YM, Zheng YT, Li X. Design, synthesis and anti-HIV-1 evaluation of hydrazide-based peptidomimetics as selective gelatinase inhibitors. Bioorgan Med Chem, 2016; 24(9):2125-36.

Yehia AT, Alzowahi FA, Kadam TA, Shaikh RU. In vitro evaluation of antimicrobial and antioxidant activity of Dragon's blood tree (Dracaena cinnabari Balf. f.) of Socotra Island (Yemen). J Coastal Life Med, 2013; 1(2):123-9.

Zabiulla, Neralagundi HS, Begum AB, Prabhakar BT, Khanum SA. Design and synthesis of diamide-coupled benzophenones as potential anticancer agents. Eur J Med Chem, 2016; 115:342-51.

\section{How to cite this article:}

Al-Ostoot FH, Mohammed YHE, Zabiulla, Kempaiah AN, Khanum SA. Synthesis, in silico study and in vitro antimicrobial evaluation of some new N-benzoyl-N'-[2-(4chloro-phenoxy)-acetyl]-hydrazides analogs. J Appl Pharm Sci, 2019; 9(07):042-049. 